\title{
Empatía y percepción del riesgo del cambio climático en estudiantes de Ciencias de la Salud
}

\section{Empathy and perception of the risk of climate change in Health Sciences students}

\author{
Alexander Salazar-Ceballos ${ }^{1}$ (D), Lidice Álvarez-Miño² (iD) \\ 1. Universidad del Magdalena. Santa Marta, Colombia. Correo: asalazar@unimagdalena.edu.co - https://orcid.org/0000-0002-0708-8792 \\ 2. Universidad del Magdalena. Santa Marta, Colombia. Correo: lalvarezm@unimagdalena.edu.co - https://orcid.org/0000-0002-1414-9442 \\ Tipología: Artículo de investigación científica y tecnológica \\ Para citar este artículo: Salazar-Ceballos A, Lídice-Álvarez M. Empatía y percepción del riesgo del cambio climático en estudiantes de ciencias de la salud. Duazary. 2020 julio - \\ septiembre; 17(3): 10 - 24. Doi: http://dx.doi.org/10.21676/2389783X.3317 \\ Recibido en febrero 11 de 2019 \\ Aceptado en julio 26 de 2019 \\ Publicado en línea en abril 18 de 2020
}

\section{RESUMEN}

Palabras clave:

cambio

climático; salud

pública;

empatía;

estudiantes del

área de la salud;

estudios

controlados

antes y

después.

Para determinar el aumento de los niveles de empatía y percepción del riesgo del cambio climático en estudiantes universitarios de la salud se realizó un estudio cuasi-experimental. La intervención fue una estrategia educativa. Se seleccionaron por conveniencia dos grupos de estudiantes de ciencias de la salud. Los niveles de empatía se midieron con la Escala de Empatía Médica de Jefferson versión estudiantes y de la Encuesta de percepción sobre riesgo al cambio climático como una amenaza para la salud humana se midieron dos ítems: índice de percepción de riesgo y conocimiento sobre los efectos en la salud por causa del cambio climático. Se encontraron diferencias entre pre y post-test $(p<0,05)$. En el pre-test ambos grupos presentaron niveles de empatía altos con un promedio de 107. Para el post-test, el grupo experimental, presentó un aumento estadísticamente significativo en los niveles de empatía. Se establecieron correlaciones significativas entre la empatía global y los factores de empatía: cuidado con compasión y toma de perspectiva; y correlación significativa entre el factor de empatía Cuidado con compasión y Conocimiento sobre los efectos en la salud por el cambio climático. Este trabajo evidenció como una estrategia educativa puede fomentar la empatía en estudiantes de la salud.

\section{ABSTRACT}

\section{Keywords:}

climate change;

public health;

empathy;

student; health

occupations;

Pre-Post Tests
To determine the increase in levels of empathy and perception of the risk of climate change in university health students, a quasi-experimental study was carried out. The intervention was an educational strategy. Two groups of health science students were selected for convenience. Empathy levels were measured using the Jefferson Medical Empathy Scale for health professions students' version and from the Climate Change Risk Perception Survey as a threat to human health two items were measured: risk perception index and knowledge about health effects because of climate change. Statistical differences were found between pre and post-test $(p<0.05)$. In the pre-test both groups presented high levels of empathy with an average of 107 . For the post-test, the experimental group presented a statistically significant increase in empathy levels. Finally, statistically significant correlations were observed between global empathy and empathy factors: compassionate care and perspective taking; and beware of compassion and knowledge about the health effects of climate change. This work showed how an educational strategy can foster empathy in health students. 


\section{INTRODUCCIÓN}

La Organización Mundial de la salud considera que el cambio climático es una amenaza para la salud pública $^{1}$; y el actual cambio climático es un fenómeno antropogénico, derivado principalmente de la quema de combustibles fósiles, como la gasolina, los cuales generan dióxido de carbono$\mathrm{CO}_{2^{-}}$, el principal gas del efecto invernadero y por consiguiente del cambio climático. Pero, además, el consumo creciente de energía y aumento de la población de los seres humanos y de sus necesidades están conduciendo a acelerar el cambio climático ${ }^{2}$.

Acorde con las predicciones, en los próximos años se espera un aumento de la temperatura ${ }^{2}$ y por consiguiente algunos de los efectos del cambio climático sobre la salud pública serán el aumento del dengue ${ }^{3}$ y la malaria ${ }^{4,5}$, cólera ${ }^{6-8}$. Además, el aumento continuado de la temperatura de los últimos años ha generado olas de calor afectando la tasa de mortalidad global, la contaminación ambiental y otras enfermedades, como las psiquiátricas ${ }^{7}$; igualmente el aumento de la temperatura genera sequías y estas generan nuevos o agravan los conflictos armados existentes, pues la lucha ya es por el acceso al agua ${ }^{9}$. Y las poblaciones más afectadas, serán las más vulnerables, es decir aquellas que viven en la extrema pobreza y con necesidades básicas insatisfechas, y entre estos se encuentran: los niños y adultos mayores (ancianos), quienes por su condición son dependientes físicamente, y su sistema inmune o está madurando como en los niños, o se está deteriorando como en los ancianos, y a estos últimos se les debe sumar las enfermedades crónicas; y las mujeres son afectadas porque son las que se desplazan grandes distancias para conseguir agua para sus familias, sobre todo en países africanos. Así estas poblaciones son, y serán, las más afectadas pero que han sido las menos responsables de la actual amenaza ${ }^{10}$.

Las predicciones para Colombia, entre 2011 y 2040, estiman que en promedio la temperatura aumentará cerca de $3^{\circ} \mathrm{C}$, y para el Caribe Colombiano se espera que se reduzcan las lluvias en un $30 \%^{11,12}$. Los efectos del cambio climático sobre en esta región no son alentadores: es la zona del país con mayor porcentaje de desertificación, pero en cambio las zonas costeras ya han tenido un aumento de los niveles del mar en cerca de 1-2 milímetros por año ${ }^{13,14}$; además de tener cerca de $57 \%$ de población en pobreza extrema ${ }^{15}$, indicando que los más pobres sufren o sufrirán los efectos del cambio climático ${ }^{1}$.

Entre los estudios de percepción sobre los riesgos del cambio climático sobre la salud de las personas en Santa Marta, se encontró que las personas vulnerables consideran que las acciones políticas disminuirían los efectos sobre la salud derivados del cambio climático ${ }^{16}$ y en otro estudio, realizado en la Universidad del Magdalena, se observó que los estudiantes de salud de semestres avanzados presentan mayor percepción del riesgo de los efectos del cambio climático sobre la salud de las personas ${ }^{17}$.

Aunque exista suficiente evidencia científica sobre las transformaciones antropogénicas del ambiente, los gobiernos, al retirarse del Acuerdo de París, toman decisiones políticas contrarias a la adaptación y mitigación del cambio climático ${ }^{18}$. Y, de hecho, existe una tendencia de escepticismo hacia la evidencia científica, argumentando que el actual cambio climático no es originado por el hombre; acorde con un reciente trabajo, se argumenta que el cambio climático se ha politizado, ya que la reducción del $\mathrm{CO}^{2}$ implica una pérdida económica, pero otros países contaminantes como China e India han optado por investigar y promover las energías alternativas y limpias ${ }^{18,19}$.

Además, se ha observado que el escepticismo hacia el cambio climático está relacionado con tener baja empatía ${ }^{20-24}$, demostrando que se deben hacer cambios a diferente nivel en el camino, por ejemplo, para avanzar hacia el logo Objetivos de Desarrollo Sostenible - ODS - 2030 25,26 como una alternativa por la salud, el bienestar y la vida. Por lo anterior, una de las rutas de investigación en esta área es conocer cómo fomentar la empatía para que ayude al cuidado del medio ambiente y por consiguiente al bienestar y salud de las personas.

Desde la evidencia científica se puede definir un perfil de las personas con mayor empatía y comprensión de los efectos del cambio climático: son mujeres, con niveles avanzados de educación, con conexión afectiva por la naturaleza, y políticamente liberales ${ }^{27-29}$. 
Los análisis desde otras perspectivas como la Orientación de la Dominancia Social (ODS), en donde se define que los individuos con alta dominancia social son los que aceptan la inequidad social entre los grupos, la jerarquía entre los grupos, como algo natural y que es moralmente aceptable $^{30}$; así desde esta perspectiva se ha evidenciado que las personas que tienen bajos niveles de empatía y altos niveles de ODS tienen menos valores medioambientales (preservación de la naturaleza) $)^{20-22}$.

La empatía se ha estudiado desde diferentes disciplinas: psicología, medicina, neurociencias e incluso desde la economía, y presentan casi una definición común: comprender y compartir los estados emocionales de los otros ${ }^{31-33}$, esta comprensión llega a ser parte de los rasgos de la personalidad y por lo tanto permite comprender por qué las personas más desfavorecidas, y todos los seres vivos, sufren los efectos del cambio climático.

En el cuidado del paciente la empatía juega un papel central en la relación entre el personal de salud y los pacientes ${ }^{23}$, y se ha observado que quienes tienen mayor empatía son: las mujeres y los estudiantes de semestres avanzados ${ }^{24}$ y que esta aumenta según el tiempo de contacto con los pacientes $^{31}$. La evidencia científica sugiere que para aumentar los niveles de empatía son necesarias las intervenciones ${ }^{31}$, aunque otros autores han encontrado que inmediatamente después de las intervenciones los niveles de empatía son altos, pero con el tiempo tienden a decaer ${ }^{32}$; y en estudiantes de varias profesiones de la salud la empatía aumentó significativamente después de implementar intervenciones específicas ${ }^{33}$.

En Colombia también se han realizado mediciones de empatía entre las profesiones de la salud encontrando que las mujeres-médicas presentan mayor interacción empática que los hombresmédicos $^{34}$ que los estudiantes de cuarto año medicina presentaron mayores niveles de empatía ${ }^{35}$, entre los estudiantes de enfermería se observó asociación estadísticamente significativa entre la empatía global con el curso académico y con el promedio académico acumulado, así como, entre estar en el lugar del paciente con el rendimiento académico ${ }^{36}$.
Desde esta perspectiva, el objetivo del presente trabajo fue observar los cambios surgidos después de una intervención educativa en salud pública, en el aumento de empatía y percepción del riesgo del cambio climático en la salud entre dos grupos de estudiantes de ciencias de la salud de la Universidad del Magdalena.

\section{MATERIALES Y MÉTODOS}

\section{Tipo de Investigación}

Se realizó un estudio cuasi-experimental con dos grupos y evaluados de forma pre-test y post-test; pero este trabajo no es un experimento porque los estudiantes no fueron seleccionados o asignados aleatoriamente a los grupos experimental o de control $^{37,38}$; el grupo experimental en empatía y percepción del riesgo del cambio climático durante los meses de febrero y mayo de 2018, en la Universidad del Magdalena. Participó de una estrategia educativa y el grupo control no recibió la intervención educativa.

\section{Participantes}

Se seleccionaron a conveniencia 38 estudiantes, distribuidos en dos grupos, uno control (estudiantes de psicología=27) y otro experimental (estudiantes de enfermería=11). El grupo experimental estuvo constituido por estudiantes del curso "salud pública", que es una electiva profesional de la Facultad de Ciencias de la Salud. El grupo control fue un grupo de estudiantes de psicología, también pertenecientes a la Facultad de Ciencias de la Salud, pero que no recibieron el curso de salud pública. Dado que los datos analizados fueron los de cada sujeto dentro de cada grupo, es decir fueron datos pareados, y no hubo asignación aleatoria de los estudiantes a los grupos, por lo tanto, se llevó a cabo la prueba de Wilcoxon, como se explicará más adelante.

\section{Intervención educativa con enfoque de los efectos del cambio climático sobre la salud pública}

Ambos grupos fueron evaluados pre y pos test. El pre-test permitió identificar condiciones similares entre grupos al inicio, frente a los niveles de empatía. La intervención duró 12 semanas con sesiones presenciales de dos horas por semana. 
El grupo control tuvo las sesiones, en las cuales se trataron temas de estadística aplicada a la vida, pero sin un énfasis específico en la salud pública. Para el grupo experimental, se desarrollaron sesiones basadas en la estrategia de salas situacionales, en las cuales se analizaron casos puntuales, a partir de la identificación y priorización de los determinantes sociales de la salud, el uso de datos estadísticos para establecer la magnitud de los riesgos en salud pública y la generación de propuestas de intervención para transformar la vida de las personas y prevenir nuevos problemas. Durante el tiempo establecido se trabajó sobre la primera dimensión del Plan Decenal de Salud Pública $^{39}$, específicamente, salud ambiental y cambio climático. Los temas sobre los cuáles se trabajó fueron: olas de calor y su relación con parto pre-término e infarto agudo al miocardio; agua y su relación con mortalidad infantil por EDA e infecciones asociadas a la atención en salud.

\section{Instrumentos}

\section{Medición de los niveles de empatía}

Para conocer los niveles de empatía se aplicó la Escala de Empatía Médica de Jefferson (EEMJ) para profesiones de la salud versión estudiantes, validada en México y adaptada a Colombia a través del criterio de jueces, aunque su aplicación inicialmente fue en estudiantes de medicina, después se desarrolló una nueva versión ampliada a las diferentes profesiones de la salud la cual tiene leves modificaciones desde la versión original ${ }^{34,35,40-}$ ${ }^{44}$. La EEMJ para las profesiones de la salud versión estudiantes consta de 20 ítems tipo Likert en una escala de 1 a 7 , donde 1 es totalmente en desacuerdo y 7 es totalmente de acuerdo; pero para evitar respuestas automáticas, la mitad de los ítems son negativos y por consiguiente la respuesta es invertida. El total del puntaje está en un rango entre 20 y 140. La EMMJ mide la empatía en tres dimensiones: toma de perspectiva, cuidado con compasión, y ponerse en el lugar del paciente. Para medir los niveles de empatía se adoptó la siguiente escala basada en los puntajes: entre 20 y 35 puntos se consideró un nivel empatía Deficiente, entre 26 y 61 puntos se consideró un nivel empatía Bajo, entre 62 y 87 se consideró un nivel empatía Intermedio, entre 88 y 113 se consideró un nivel empatía Sobresaliente, y entre 114 y 140 se consideró un nivel empatía alto ${ }^{43}$.

Para los análisis estadísticos la EMMJ se dividió en los tres factores, donde toma de perspectiva tiene puntajes entre 10 y 70 y comprende los ítems $2,4,5,9,10,13,15,16,17$ y 20 ; cuidado con compasión tiene puntajes entre 8 y 56 y comprende los ítems $1,7,8,11,12,14,18$ y 19 ; y ponerse en el lugar del paciente tiene puntajes entre 2 y 14 y comprende los ítems 3 y $6^{44}$. Con los datos suministrados se hicieron los análisis estadísticos correspondientes para el tipo de información, sin embargo, como limitante se reconoce que al tratarse de una muestra no aleatoria sus resultados no pueden ser extrapolados ${ }^{45}$.

\section{Medición de los niveles de conocimiento del cambio climático y sus efectos sobre la salud pública}

Para conocer los niveles de conocimiento del cambio climático y sus efectos sobre la salud pública se aplicó el instrumento "Encuesta de percepción sobre riesgo al cambio climático como una amenaza para la salud humana" tomado y adaptado de DeBono et $a l^{46}$, validado en Colombia, donde la variable Índice de Percepción de Riesgo tiene un $\alpha$ de Cronbach de 0,77 , y la variable Conocimiento sobre los efectos en la salud por causa del cambio climático tiene una confiabilidad Kuder-Richardson de $0,40^{16}$.

En el Índice de percepción de riesgo se abordan afirmaciones sobre el conocimiento del impacto del cambio climático, sobre el modo de vida de las personas y presenta cinco respuestas tipo Likert que van desde No probable, valorado con 1, hasta muy probable, valorado con 5 . Algunas de las afirmaciones de este índice son: En todo el mundo se producirá escasez de agua como consecuencia del cambio climático, y en Santa Marta habrá escasez de agua como consecuencia del cambio climático. Y el ítem Conocimiento sobre los efectos en la salud por causa del cambio climático incluye aspectos como las enfermedades infecciosas, asma, alergias, cáncer, alteraciones cardiovasculares, entre otros; con respuestas de tipo booleano: Sí o No. En el artículo original se estableció una valoración de las respuestas así: un punto fue adicionado por cada respuesta correcta y un punto fue deducido por cada respuesta incorrecta ${ }^{46}$. 


\section{Análisis estadísticos}

Se realizaron inicialmente análisis de estadística descriptiva con promedios y desviaciones estándar, medianas y cuartiles. De los análisis se generaron gráficos de box-plot para identificar las diferencias relativas entre los grupos control y experimental. Los resultados antes y después, tanto de los grupos control y experimental, fueron comparados con la prueba de Wilcoxon; se utilizó la prueba no paramétrica de Wilcoxon, porque se analizaron los datos pareados (pre-test y post-test) de cada sujeto dentro de cada grupo y no hubo seleción/asignación aleatoria de los estudiantes a los grupos ${ }^{37,38}$. Se realizó la correlación de Spearman para identificar relación entre los niveles de empatía global con los diferentes factores y con el conocimiento de los efectos del cambio climático sobre la salud pública entre los estudiantes. Para la interpretación de la correlación de Spearman se consideró la asociación entre dos variables ordinales aleatorias con valores entre -1 y 1 , donde cero indicar ausencia de relación. Los resultados de la prueba de rangos por Wilcoxon y correlación por Spearman fueron considerados estadísticamente significativos cuando $p<0,05$. Los análisis estadísticos se realizaron con los softwares de Excel, Epi-Info y R.

\section{Declaración sobre aspectos éticos}

Este estudio adaptó las normas establecidas en la Declaración de Helsinki de 1975 y la Resolución 8430 de 1993 del Ministerio de Salud de Colombia para la investigación con seres humanos. A todos los participantes se les explicó el proyecto y se les presentó el consentimiento informado. Todos los datos corresponden a estudiantes que aceptaron participar porque comprendieron el estudio $y$ firmaron el consentimiento informado, en el cual se garantizó confidencialidad y derecho a la no participación o retiro.

\section{RESULTADOS}

El grupo control estuvo comprendido por 27 estudiantes de psicología, de los cuales el 63\%(17) fueron mujeres y la media de la edad fue de 19 años ( $D E=1,81)$. El grupo de intervención estuvo comprendido por 11 estudiantes de enfermería, de los cuales el $73 \%$ (8) fueron mujeres y la media de la edad fue de 21 años (DE $=4,76)$.

En el grupo control, los resultados de la escala de empatía (Tabla 1), tanto en sus 3 factores como en la empatía global, no presentó diferentes significativas entre los grupos pre y post-test. Se puede observar que el promedio de la empatía global estuvo entre $106(\mathrm{DE}=10,83)$ y $107(\mathrm{DE}=$ $15,49)$, promedio considerado sobresaliente ${ }^{43}$.

Tabla 1. Resultados de empatía y sus factores en el grupo control comparando los pre-test y post-test.

\begin{tabular}{|c|c|c|c|c|c|c|c|c|}
\hline \multicolumn{9}{|c|}{ Grupo control } \\
\hline & \multicolumn{2}{|c|}{ Toma de perspectiva } & \multicolumn{2}{|c|}{$\begin{array}{l}\text { Cuidado con } \\
\text { compasión }\end{array}$} & \multicolumn{2}{|c|}{$\begin{array}{c}\text { Ponerse en el } \\
\text { lugar del } \\
\text { paciente }\end{array}$} & \multicolumn{2}{|c|}{ Empatía global } \\
\hline & Pre & Post & Pre & Post & Pre & Post & Pre & Post \\
\hline Media & 56,9 & 58,96 & 42,89 & 39,96 & 7,78 & 7,7 & 107,59 & 106,67 \\
\hline Mediana & 56 & 60 & 45 & 42 & 7 & 8 & 107 & 108 \\
\hline Moda & 55 & 62 & 41 & 42 & 6 & 7 & 103 & 108 \\
\hline Desviación estándar & 7,6 & 7 & 6,57 & 9,38 & 2,98 & 2,6 & 10,83 & 15,49 \\
\hline Mínimo & 38 & 43 & 28 & 17 & 2 & 2 & 85 & 73 \\
\hline Máximo & 70 & 70 & 52 & 53 & 14 & 13 & 128 & 127 \\
\hline $\begin{array}{c}\text { Prueba de Wilcoxon, } \\
\text { valor } Z(p)\end{array}$ & \multicolumn{2}{|c|}{$Z=-1,547(p>0,05)$} & \multicolumn{2}{|c|}{$Z=-1,828(p>0,05)$} & \multicolumn{2}{|c|}{$\mathrm{Z}=-\frac{-0,324(p>}{0,05)}$} & \multicolumn{2}{|c|}{$Z=-0,042(p>0,05)$} \\
\hline
\end{tabular}


En el grupo experimental el nivel empatía global antes del tratamiento fue de $108(D E=12,63)$ y después del tratamiento fue de $116(\mathrm{DE}=8,85)$ puntos; se considera que un puntaje mayor de 114 puntos es un nivel de empatía alto ${ }^{43}$. En la Figura 1, se puede observar con mayor claridad la comparación entre los resultados del pre y pos-test sin cambios estadísticamente significativos para el grupo control.

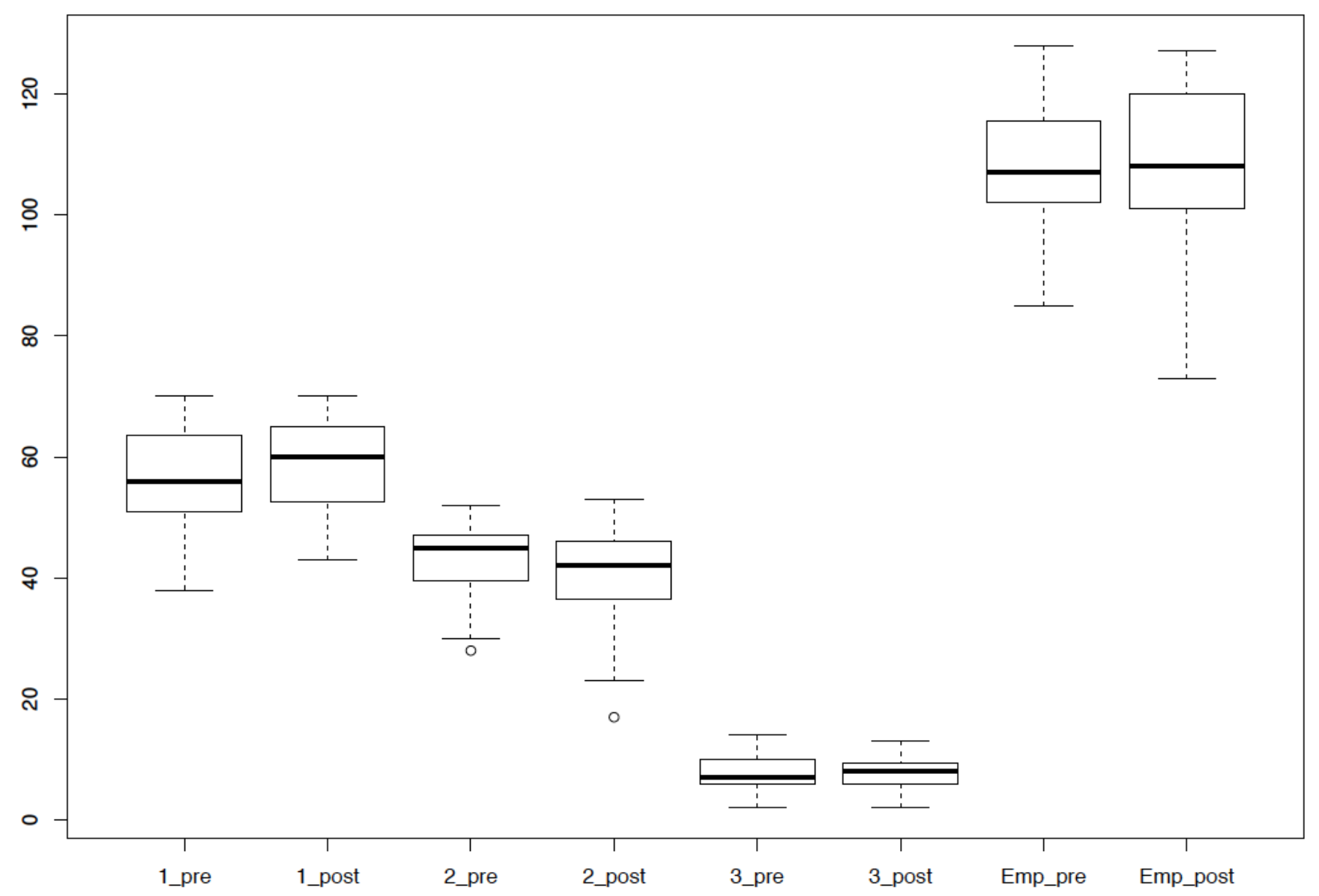

Figura 1. Boxplots de los resultados de empatía y sus factores en el grupo control comparando los pre-test y post-test.

1_pre: Toma de perspectiva, pre-test; 1_post: Toma de perspectiva, pre-test; 2_pre: Cuidado con compasión, pre-test; 2_post: Cuidado con compasión, post; 3_pre: Ponerse en el lugar del paciente, pre-test; 3_post-test: Ponerse en el lugar del paciente, post-test; Emp_pre: Empatía, pre-test; Emp_post: Empatía, post-test

En el grupo experimental se observó una diferencia estadísticamente significativa en los resultados de la empatía global en la comparación pre-test (108) y post-test (116), la prueba de Wilcoxon fue de 2,39 con un valor de $p<0,05$. Y en el análisis por factores de la empatía se observó una diferencia estadísticamente significativa en los resultados del factor Cuidado con compasión en la comparación pre y post-test, la prueba de Wilcoxon fue de $-2,24$ con un valor de $\mathrm{p}<0,05$ (Tabla 2 ). 
Tabla 2. Resultados de empatía y sus factores en el grupo experimental comparando los pre-test y post-test.

\begin{tabular}{|c|c|c|c|c|c|c|c|c|}
\hline \multicolumn{9}{|c|}{ Grupo experimental } \\
\hline & \multicolumn{2}{|c|}{ Toma de perspectiva } & \multicolumn{2}{|c|}{ Cuidado con compasión } & \multicolumn{2}{|c|}{$\begin{array}{c}\text { Ponerse en el } \\
\text { lugar del } \\
\text { paciente }\end{array}$} & \multicolumn{2}{|c|}{ Empatía global } \\
\hline & Pre & Post & Pre & Post & Pre & Post & Pre & Post \\
\hline Media & 60,55 & 61,45 & 40,36 & 46,91 & 7,09 & 7,73 & 108 & 116,09 \\
\hline Mediana & 61 & 62 & 41 & 46 & 8 & 8 & 108 & 115 \\
\hline Moda & 65 & $\# N / A$ & 44 & 44 & 8 & 8 & 112 & 107 \\
\hline Desviación estándar & 5,32 & 5,91 & 9,95 & 5,28 & 2,34 & 2,15 & 12,63 & 8,85 \\
\hline Mínimo & 52 & 53 & 26 & 35 & 3 & 4 & 90 & 104 \\
\hline Máximo & 67 & 70 & 56 & 54 & 12 & 12 & 129 & 129 \\
\hline $\begin{array}{l}\text { Prueba de Wilcoxon, valor } \\
\qquad \mathrm{Z}(p)\end{array}$ & \multicolumn{2}{|c|}{$\begin{array}{l}\mathrm{Z}=0,8447 \\
(p>0,05)\end{array}$} & \multicolumn{2}{|c|}{$\begin{array}{c}\mathrm{Z}=-2,24 \\
(p<0,05)^{*}\end{array}$} & \multicolumn{2}{|c|}{$\begin{aligned} Z= & -0,8452(p \\
& >0,05)\end{aligned}$} & \multicolumn{2}{|c|}{$\begin{array}{c}\mathrm{Z}=-2,39 \\
(p<0,05)^{*}\end{array}$} \\
\hline
\end{tabular}

${ }^{*} \mathrm{p}<0,05$ = estadísticamente significativo.

En la Figura 2 se observa la comparación entre antes y después del tratamiento en el grupo de intervención y se nota que la mediana factor Cuidado con compasión, factor 2 , es mayor después del tratamiento. Además, los resultados de empatía global fueron mayores después del tratamiento, diferencias significativas que fueron confirmadas a través de la prueba de Wilcoxon.

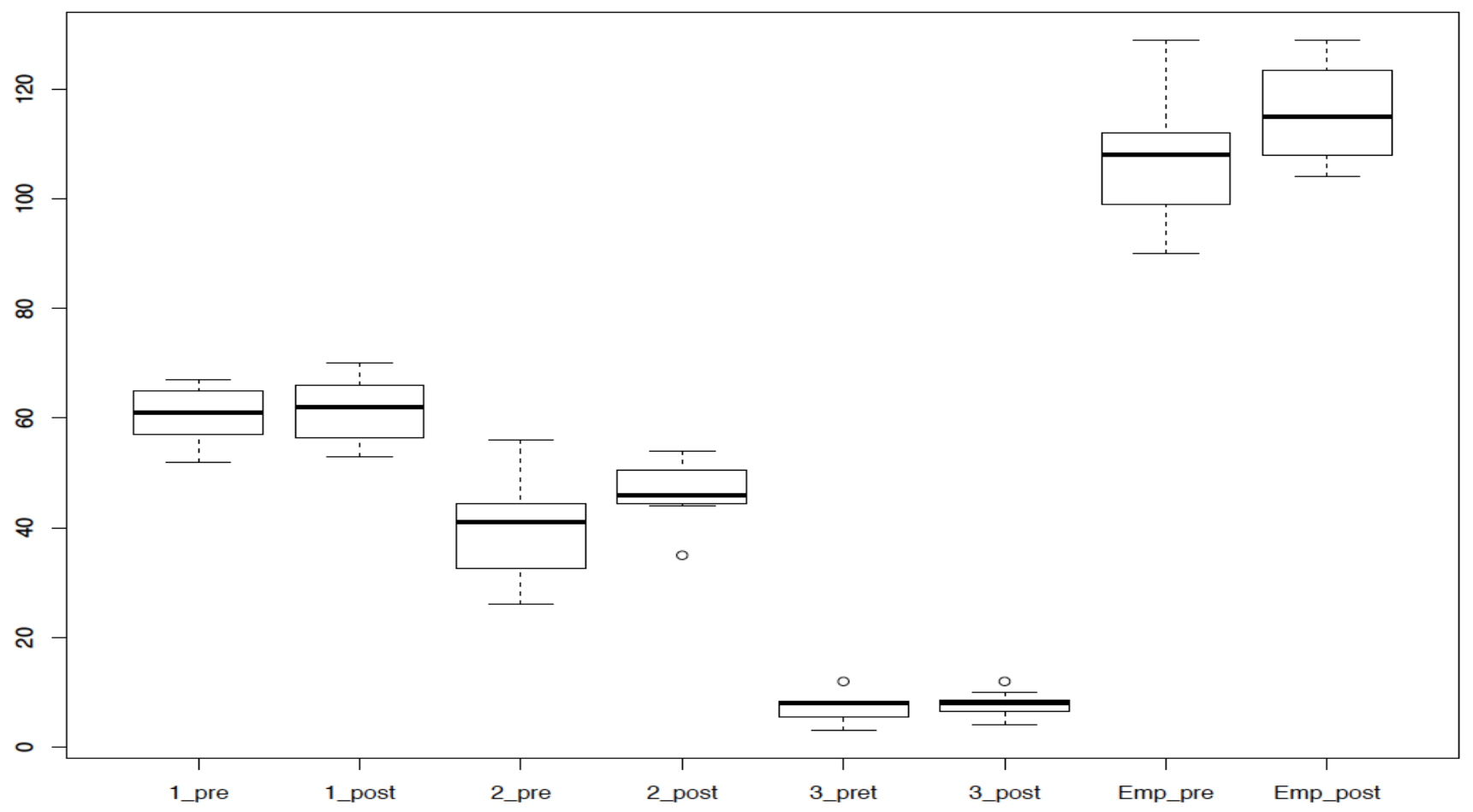

Figura 2. Boxplots de los resultados de empatía y sus factores en el grupo experimental comparando los pre-test y posttest.

1_pre: Toma de perspectiva, pre-test; 1_post: Toma de perspectiva, pre-test; 2_pre: Cuidado con compasión, pre-test; 2_post: Cuidado con compasión, post; 3_pre: Ponerse en el lugar del paciente, pre-test; 3_post-test: Ponerse en el lugar del paciente, post-test; Emp_pre: Empatía, pre-test; Emp_post: Empatía, post-test. 
En el grupo control, a nivel de los resultados del Índice de percepción de riesgo y del Conocimiento sobre los efectos en la salud por causa del cambio climático no se observaron diferencias estadísticamente significativas entre los resultados antes y después (Tabla 3).

Tabla 3. Resultados en el grupo control del Índice de percepción de riesgo y del Conocimiento sobre los efectos en la salud por causa del cambio climático.

\begin{tabular}{|c|c|c|c|c|}
\hline \multicolumn{5}{|c|}{ Grupo control } \\
\hline & \multicolumn{2}{|c|}{ Índice de percepción de riesgo } & \multicolumn{2}{|c|}{$\begin{array}{c}\text { Conocimiento sobre los efectos en la salud } \\
\text { por el cambio climático }\end{array}$} \\
\hline & Pre & Post & Pre & Post \\
\hline Media & 25,2 & 26,3 & 0,4 & 0,9 \\
\hline Mediana & 25 & 26 & 1 & 1 \\
\hline Moda & 30 & 30 & -1 & 3 \\
\hline Desviación estándar & 3,7 & 2,9 & 1,8 & 2 \\
\hline Mínimo & 17 & 19 & -3 & -3 \\
\hline Máximo & 30 & 30 & 4 & 3 \\
\hline Prueba de Wilcoxon, valor Z ( $p)$ & \multicolumn{2}{|c|}{$Z=-1,85(p>0,05)$} & \multicolumn{2}{|c|}{$Z=-1,15(p>0,05)$} \\
\hline
\end{tabular}

En el grupo experimental, a nivel de los resultados del Indice de percepción de riesgo se observaron diferencias estadísticamente significativas $(Z=-$ 1,987, $p<0,05)$ entre los resultados antes $y$ después. Pero a nivel Conocimiento sobre los efectos en la salud por causa del cambio climático no se observaron diferencias estadísticamente significativas entre los resultados antes y después (Tabla 4).

Tabla 4. Resultados en el grupo experimental del Índice de percepción de riesgo y del conocimiento sobre los efectos en la salud por causa del cambio climático. Fuente: elaboración propia.

\begin{tabular}{|c|c|c|c|c|}
\hline \multicolumn{5}{|c|}{ Grupo experimental } \\
\hline & \multicolumn{2}{|c|}{ Índice de percepción de riesgo } & \multicolumn{2}{|c|}{$\begin{array}{l}\text { Conocimiento sobre los efectos en la salud } \\
\text { por el cambio climático }\end{array}$} \\
\hline & Pre & Post & Pre & Post \\
\hline Media & 26 & 28,27 & $-0,36$ & 2,64 \\
\hline Mediana & 27 & 29 & 1 & 3 \\
\hline Moda & 24 & 30 & -3 & 3 \\
\hline Desviación estándar & 3,38 & 2,49 & 2,46 & 0,81 \\
\hline Mínimo & 20 & 22 & -3 & 1 \\
\hline Máximo & 30 & 30 & 3 & 3 \\
\hline $\begin{array}{l}\text { Prueba de Wilcoxon, valor } \\
\qquad \mathrm{Z}(p)\end{array}$ & \multicolumn{2}{|c|}{$Z=-1,987(p<0,05)^{*}$} & \multicolumn{2}{|c|}{$Z=-2,52$, sin cálculo del valor $p$} \\
\hline
\end{tabular}

$* p<0,05:$ estadísticamente significativo. 
Se realizaron análisis de correlación de Spearman con los resultados post-test en el grupo experimental. Se observó correlación estadísticamente significativa entre el factor de empatía Cuidado con compasión y la empatía global $\left(r_{s}=0,86, p<0,05\right)$ y con Conocimiento sobre los efectos en la salud por el cambio climático $\left(r_{s}=0,63, p<0,05\right)$; igualmente se observó correlación estadísticamente significativa entre el factor de empatía Toma de perspectiva y la empatía global $\left(r_{s}=0,67, p<0,05\right)$ (Tabla 5).

Tabla 5. Correlación de Spearman en el grupo experimental.

\begin{tabular}{|c|c|c|c|c|c|c|}
\hline & $\begin{array}{c}\text { Toma de } \\
\text { perspectiva }\end{array}$ & $\begin{array}{l}\text { Cuidado con } \\
\text { compasión }\end{array}$ & $\begin{array}{l}\text { Ponerse } \\
\text { en el } \\
\text { lugar del } \\
\text { paciente }\end{array}$ & $\begin{array}{l}\text { Empatía } \\
\text { global }\end{array}$ & $\begin{array}{c}\text { Índice de } \\
\text { percepción de } \\
\text { riesgo }\end{array}$ & $\begin{array}{l}\text { Conocimiento } \\
\text { sobre los efectos } \\
\text { en la salud por el } \\
\text { cambio climático }\end{array}$ \\
\hline Toma de perspectiva & 1 & & & & & \\
\hline $\begin{array}{l}\text { Cuidado con } \\
\text { compasión }\end{array}$ & 0,2746060 & 1 & & & & \\
\hline $\begin{array}{c}\text { Ponerse en el lugar } \\
\text { del paciente }\end{array}$ & 0,2027844 & 0,5939819 & 1 & & & \\
\hline Empatía global & $0,6758061^{*}$ & $0,8666690 *$ & $\begin{array}{c}0,421314 \\
4\end{array}$ & 1 & & \\
\hline $\begin{array}{c}\text { Indice de percepción } \\
\text { de riesgo }\end{array}$ & 0,2819773 & $-0,1178989$ & $\begin{array}{c}- \\
0,038766 \\
5\end{array}$ & 0,1728414 & 1 & \\
\hline $\begin{array}{l}\text { Conocimiento sobre } \\
\text { los efectos en la } \\
\text { salud por el cambio } \\
\text { climático }\end{array}$ & 0,0745356 & $0,6379170 *$ & $\begin{array}{c}0,377866 \\
3\end{array}$ & 0,5241371 & $-0,07836977$ & 1 \\
\hline
\end{tabular}

\section{DISCUSIÓN}

El objetivo del presente trabajo fue identificar, después de una intervención educativa, el aumento de niveles de empatía y percepción del riesgo de los efectos de la salud al cambio climático entre los estudiantes de salud de la Universidad del Magdalena. En los análisis de los resultados se observó que el grupo experimental presentó aumento estadísticamente significativo de los niveles de empatía, y además se observó correlación estadísticamente significativa entre el factor de empatía Cuidado con compasión y Conocimiento sobre los efectos en la salud por el cambio climático.

En este trabajo, en ambos grupos, control y experimental, durante el pre-test se observaron puntajes sobresalientes de empatía global ${ }^{43}$ con un puntaje promedio de 107. Durante el post-test el grupo experimental presentó un aumento estadísticamente significativo de los niveles de empatía, con un promedio de 116. Igualmente, en el grupo experimental se observó un aumento estadísticamente significativo en los niveles del factor de empatía Cuidado con compasión y en el Índice de percepción de riesgo. Finalmente se observaron correlaciones estadísticamente significativas entre la empatía global y los factores de empatía Cuidado con compasión y Toma de perspectiva; igualmente entre el factor de empatía Cuidado con compasión y Conocimiento sobre los efectos en la salud por el cambio climático.

En este trabajo se encontró que los niveles de empatía entre los dos grupos del estudio tuvieron un puntaje empatía sobresaliente, con un rango entre 106 y 108; y en el post-test se observó un aumento en el grupo tratamiento, el cual tuvo un promedio de 116 puntos, lo que se considera como un nivel de empatía alto, siendo este la escala 
mayor según un trabajo previo donde un puntaje entre 88 y 113 es sobresaliente, y un puntaje entre 114 y 140 es alto ${ }^{43}$. Así, el promedio de puntaje sobresaliente en ambos grupos en el pre-test en este trabajo está acorde con un trabajo llevado entre estudiantes de medicina en México, donde obtuvieron un promedio de 113 puntos ${ }^{43}$. También los puntajes de la presente investigación son similares a los obtenidos entre estudiantes de medicina en la Universidad del Norte, en la ciudad de Barranquilla en Colombia, donde el promedio entre los diferentes años de la carrera estuvo entre 106 y 111 puntos, es decir con un promedio sobresaliente ${ }^{35}$.

Los cambios en el promedio del nivel de empatía en el grupo de intervención antes y después fueron estadísticamente significativos, lo que sugiere que las actividades académicas realizadas fueron las adecuadas durante las 12 semanas de estudio. En un estudio previo realizado en Australia, con estudiantes del área de la salud también se observaron cambios estadísticamente significativos en el nivel de empatía en el grupo que recibió la intervención, e igual que en este caso, el tiempo de intervención fue de 12 semanas $^{47}$. En otro estudio, donde se llevó a cabo un diseño experimental en dos grupos, el de control con 31 enfermeras y el experimental con 17 enfermeras, y se les realizó pre y post-test, y se observó que el grupo experimental obtuvo el mayor puntaje en la escala de empatía; las sesiones de intervención fueron 5 durante 5 semanas, cada sesión fue de 4 horas ${ }^{31}$. Estos estudios sugieren que el entrenamiento aumenta los niveles de empatía.

Las actividades de intervención dirigidas al incremento y mantenimiento de los niveles de empatía entre los profesionales de la salud es un área de investigación que aborda más allá de los estudios transversales. De ahí se ha observado que tiempo después de la intervención los niveles de empatía tienden a disminuir, entre las variables explicativas se encuentran el inicio de la práctica clínica y la falta de habilidades de comunicación ${ }^{48}$, por lo tanto, otro interrogante de investigación sería ¿Por qué no se mantienen esos altos niveles de empatía después del tratamiento?

En este trabajo, a nivel del grupo de intervención, se observaron correlaciones estadísticamente significativas entre la empatía global y los factores de empatía cuidado con compasión y Toma de perspectiva, es decir que estos fueron los dos factores de la empatía que más influyeron en el aumento de los niveles de empatía global; pero, al análisis comparativo en las medias entre los análisis pre-test y post-test el único factor que mostró un aumento estadísticamente significativo fue el factor cuidado con compasión. Y fue precisamente este el único factor de empatía que se relacionó estadísticamente con el ítem de Conocimiento sobre los efectos en la salud por el cambio climático.

El factor de empatía cuidado con compasión comprende aspectos relacionados como los sentimientos, emociones y experiencias personales del paciente. Es decir, son aspectos con los cuales los estudiantes se pueden haber sentido identificados al estar frente a los pacientes, ya que la compasión debería hacer parte de la calidad del cuidado de la salud de los pacientes, y en algunos países, como Nueva Zelanda, han impulsado el cuidado con compasión como un derecho de los pacientes ${ }^{49}$. La compasión es una conexión con quien está sufriendo y se acompaña del acto de subsanar el evento del sufrimiento ${ }^{50}$.

Debido a la intervención educativa se observó un aumento, medido a través de dos instrumentos unificados, los niveles de Índice de percepción del riesgo y el Conocimiento sobre los efectos sobre la salud por el cambio climático, aunque solo el Índice de percepción del riesgo mostró cambios estadísticamente significativos. Estos logros son un avance significativo pues se está contribuyendo a la formación de profesionales de la salud con empatía y cuidado hacia el medio ambiente; igualmente en percibir los riesgos sobre la salud derivados del medio ambiente. Estos cambios en los comportamientos se espera que tendrán efectos a largo plazo en la disminución de los efectos del cambio climático sobre la salud pública ${ }^{51}$.

En este trabajo de intervención educativa en salud pública dirigido a observar los efectos del cambio climático sobre la salud pública se observó una la relación entre los niveles de empatía y los efectos sobre la salud del cambio climático, indicando la necesidad de cuidar el medio ambiente; y es un camino que apenas comienza, pues la literatura 
también da cuenta de personas que tienen bajos niveles de empatía y que niegan los efectos del cambio climático ${ }^{20-22}$, así como que los niveles de negación y escepticismo varían geográficamente ${ }^{52}$. Estas negaciones o baja percepción y creencias del riesgo del cambio climático se presentan a pesar de la evidencia científica ${ }^{2,9,53}$.

Otros trabajos que abordan otras perspectivas como la Orientación de la Dominancia Social (ODS), la cual se refiere a que los individuos que tienen alta dominancia social son aquellos que aceptan la inequidad social entre los grupos, la jerarquía entre los grupos, como algo natural y que es moralmente aceptable ${ }^{30}$, han permitido evidenciar que quienes tienen bajos niveles de empatía y altos niveles de ODS tienen menos valores medioambientales (preservación de la naturaleza) 20-22,54.

En conclusión, la habilidad de la empatía y de su relación con los efectos del cambio climático sobre la salud pública es un área que debe ser abordada desde la formación de los futuros profesionales de la salud. Las limitaciones del presente estudio se inscriben principalmente en la selección no probabilística de los grupos y la pérdida de sujetos entre el pre y post en el grupo de intervención. Así se sugiere que futuros estudios tengan un diseño muestral estadísticamente representativo. Igualmente, se pueden abordar otras perspectivas, como la Orientación de la Dominancia Social, para identificar variables que influyen en los niveles de empatía y de su relación con los efectos sobre la salud por el cambio climático.

\section{DECLARACIÓN SOBRE CONFLICTO DE INTERESES}

Este estudio no presentó conflicto de interés con ninguna parte involucrada.

\section{CONTRIBUCIÓN DE LOS AUTORES}

Primer autor: diseño metodológico, análisis estadístico y elaboración final del manuscrito.

Segundo autor: diseño metodológico, análisis estadístico y elaboración final del manuscrito.

\section{REFERENCIAS BIBLIOGRÁFICAS}

1. World Health Organization [Internet]. Protecting health from climate change: connecting science, policy and people. 2009. [Actualizado 11 Dic 2010; Citado 6 Mar 2017]. Disponible en: http://www.who.int/globalchange/publication s/reports/9789241598880/en/

2. Intergovernmental Panel on Climate Change. Climate change 2007: Synthesis Report. Contribution of Working Group II to the Fourth Assessment Report of the Intergovernmental Panel on Climate Change [Internet]. Geneva, Switzerland: Cambrige University Press; 2007. [Citado 15 Jun 2018]. Disponible en: http://www.ipcc.ch/publications_and_data/pu blications_ipcc_fourth_assessment_report_sy nthesis_report.htm

3. Hess JJ, McDowell JZ, Luber G. Integrating Climate Change Adaptation into Public Health Practice: Using Adaptive Management to Increase Adaptive Capacity and Build Resilience. Environ Health Perspect. 2012; 120: $\quad 171-9 . \quad$ Doi: http://doi: 10.1289/ehp.1103515

4. Caminade $C$, Kovats $S$, Rocklov J, Tompkins A, Morse A, Colón-González, Stenlund H, Martens Lloyd S. Impact of climate change on global malaria distribution. PNAS. 2014; 111 (9):3286-3291. Doi: https://doi.org/10.1073/pnas.1302089111

5. Olson S, Gangnon R, Elguero E, Durieux L, Guégan F, Foley J, and Patz J. Links between Climate, Malaria, and Wetlands in the Amazon Basin. Emerg Infect Dis. 2009; 15(4):659-662. Doi: https://doi:10.3201/eid1504.080822

6. Lama J, Seas C, León-Barúa R, Gotuzzo E, Sack R. Environmental temperatura, cholera, and acute diarrhoea in adults in Lima, Peru. J Health Popul Nutr. 2004; 22 (4):399-403. Disponible en: https://www.ncbi.nlm.nih.gov/pubmed/15663 172

7. Franchini $M$, Mannuccio P. Impact on human health of climate changes. European Journal of Internal Medicine. 2015; 26: 1-5. Doi: 
http://dx.doi.org/10.1016/j.ejim.2014.12.008

8. Akil L, Ahmad HA, Reddy RS. Effects of climate change on Salmonella infections. Foodborne Pathog Dis. 2014;11(12):974-80. Doi: https://doi: 10.1089/fpd.2014.1802

9. Haines A, Kovats RS, Campbell-Lendrumb D, Corvalan $\mathrm{C}$. Climate change and human health: Impacts, vulnerability and public health. Public Health. 2006; 120:585-6. Disponible en: https://www.ncbi.nlm.nih.gov/pubmed/16798 393

10. Oloukoi G, Bob U, Jaggernath J. Perception and trends of associated health risks with seasonal climate variation in Oke-Ogun region, Nigeria. Health \& Place. 2014; 25: 47-55. Doi: http://dx.doi.org/10.1016/j.healthplace.2013. 09.009 .

11. Pabón JD. Cambio climático en Colombia: tendencias en la segunda mitad del siglo XX y escenarios posibles para el siglo XXI. Rev. Acad. Colomb. Cienc. 2012; 36 (139): 261-278. Disponible en: http://www.scielo.org.co/scielo.php?script=sci _arttext\&pid=S0370-39082012000200010

12. García M, Piñeros A, Bernal F, Ardila E. Variabilidad climática, cambio climático y el recurso hídrico en Colombia. Revista de Ingeniería. 2012; 36: 60-64. Disponible en: https://ojsrevistaing.uniandes.edu.co/ojs/inde x.php/revista/article/view/136

13. Olivero-Verbel J. Colombia: Environmental Health Issues. En: Reference Module in Earth Systems and Environmental Sciences, from Encyclopedia of Environmental Health. 2011:740-754. Doi: https://doi.org/10.1016/B978-0-444-522726.00395-0

14. Vallejo J. A Regional Strategy for Water Resource Management in the Caribbean Region of Colombia. Tropical Resources Bulletin. 2011; 30: 28-35. Disponible en: http://environment.yale.edu/tri/publications/t ropical-resources-volume-30/

15. Calderon A, Harris J, Kirsch P. Health interventions used by mayor resource companies operating in Colombia. Resources
Policy. 2016; 47:187-197. Doi: http://dx.doi.org/10.1016/j.resourpol.2015.02 .003

16. Salazar-Ceballos A, Freyle N, Tamara G, Álvarez-Miño L. Percepción sobre riesgo al cambio climático como una amenaza para la salud humana, Taganga, Santa Marta, 2014. Revista Luna Azul. 2016; 43: 102- 127. Doi: http://dx.doi.org/10.17151/luaz.2016.43.6.

17. Salazar-Ceballos A, Álvarez-Miño L, MuñozSánchez EP, Carreño-Orozco JD, RodríguezCholes BH. Percepción del riesgo al cambio climático y sus efectos sobre la salud y enfermedades infecciosas en estudiantes universitarios, Santa Marta, Colombia, 2011. Rev Cuid. 2014; 5(1): 613-22. Disponible en: http://www.scielo.org.co/scielo.php?pid=S221 6-

09732014000100005\&script=sci_abstract\&tln $\mathrm{g}=\mathrm{es}$.

18. Samet JM, Woodward A. National Government Denial of Climate Change and State and Local Public Health Action in a Federalist System. American Journal of Public Health [Internet]. 2018 Apr [cited 2019 Sep 14];108(S2): S112-3. Disponible en : https://www.ncbi.nlm.nih.gov/pubmed/29698 096

19. Van Boven L, Ehret PJ, Sherman DK. Psychological Barriers to Bipartisan Public Support for Climate Policy. Perspectives on psychological science: a journal of the Association for Psychological Science [Internet]. 2018 [cited 2019 Sep 14];13(4):492-507. Disponible en: https://www.ncbi.nlm.nih.gov/pubmed/29961 412

20. Häkkinen K, Akrami N. Ideology and climate change denial. Personality and Individual Differences. 2014; 70: 62-65. Doi: https://doi.org/10.1016/j.paid.2014.06.030

21. Jylhä KM, Akrami N. Social dominance orientation and climate change denial: The role of dominance and system justification. Personality and Individual Differences. 2015; 86:108-111. Doi: https://doi:10.1016/j.paid.2015.05.041 
22. Jylhä KM, Cantal C, Akrami N, Milfont TL. Denial of anthropogenic climate change: Social dominance orientation helps explain the conservative male effect in Brazil and Sweden. Personality and Individual Differences. 2016; 98:184-187.

Doi: http://dx.doi.org/10.1016/j.paid.2016.04.020

23. Del Canale $S$, Louis D, Maio V, Wang X, Rossi G, Hojat M, Gonnella J. The Relationship Between Physician Empathy and Disease Complications: An Empirical Study of Primary Care Physicians and Their Diabetic Patients in Parma, Italy. Acad Med. 2012; 87:1243-1249. Disponible en: https://www.ncbi.nlm.nih.gov/pubmed/22836 852

24. Urday S, Ugald R, Arancibia Z, Díaz VP. Evaluación de los niveles de orientación empática en estudiantes de odontología de la Universidad Finis Terrae de Santiago, Chile. Rev. Clin. Periodoncia Implantol. Rehabil. Oral. 2013; 6(3): 130-133. Doi: http://dx.doi.org/10.4067/S071901072013000300006

25. Sachs JD, Schmidt-Traub G, Mazzucato $M$, Messner D, Nakicenovic N, Rockström J. Six Transformations to achieve the Sustainable Development Goals. Nature Sustainability. 2019. Doi: https://doi.org/10.1038/s41893019-0352-9

26. Schmidt-Traub G. Six Transformations to achieve the Sustainable Development Goals (SDGs) [Internet]. Nature Research Sustainability Community. Springer Nature; 2019 [cited 2019 Sep 14]. Disponible en: https://sustainabilitycommunity.nature.com/u sers/276024-guido-schmidttraub/posts/52751-six-transformations-toachieve-the-sustainable-development-goalssdgs

27. Markowitz E, Goldberg L, Ashton M, Lee K. Profiling the "pro-environmental individual": a personality perspective. J Pers. 2012; 80 (1):81-111. Doi: https://doi.org/10.1111/j.14676494.2011.00721.x
28. Christov-Moore L, Simpson E, Coudé G, Grigaityte K, Lacoboni M, y Ferrari P. Empathy: gender effects in brain and behavior. Neurosci Behavior Rev. 2014; 46(Pt4); 604-627. Doi: https://doi:10.1016/j.neubiorev.2014.09.001

29. Wheeler H, Quinn C. Can Facebook aid sustainability? An investigation of empathy expression within the Humans of New York Blog. Sustainability. 2017; 9(6):1005. Doi: https://doi.org/10.3390/su9061005

30. Pratto F, Sidanius, J, Stallworth LM, Malle BF. Social dominance orientation: A personality variable predicting social and political attitudes. Journal of Personality and Social Psychology. 1994; 67(4): 741-763. Disponible en:

https://dash.harvard.edu/bitstream/handle/1/ 3207711/sidanius_socialdominanceorientation .pdf?sequence $=1$

31. Kahriman I, Nural N, Arslan U, Topbas M, Can G, Kasim S. The Effect of Empathy Training on the Empathic Skills of Nurses. Iran Red Crescent Med J. 2016; 18(6): e24847. Doi: http//doi.org/10.5812/ircmj.24847

32. Nunes P, Williams S, Sa B, Stevenson K. A study of empathy decline in students from five health disciplines during their first year of training. International Journal of Medical Education. 2011; 2:12-17. Doi: https://doi:10.5116/ijme.4d47.ddb0

33. Williams B, Brown T, McKenna L, Palermo C, Morgan P, Nestel D, et al. Student empathy levels across 12 medical and health professions: an interventional study. Journal of Compassionate Health Care. 2015; 2:4. Doi: https://doi.org/10.1186/s40639-015-0013-4

34. Alcorta-Garza A, San-Martín M, DelgadoBolton R, Soler-González J, Roig $\mathrm{H}$, Vivanco L. Cross-Validation of the Spanish HP-Version of the Jefferson Scale of Empathy Confirmed with Some Cross-Cultural Differences. Front. Psychol. 2016; 7:1002. Doi: https://doi:10.3389/fpsyg.2016.01002

35. Alonso LM, Caro S, Erazo AM, Díaz V. Evaluación de la orientación empática en estudiantes de medicina de la Universidad del 
Norte, Barranquila (Colombia). Salud Uninorte. 2013; 29 (1): 22-33. Disponible en: ttp://rcientificas.uninorte.edu.co/index.php/sa lud/article/viewArticle/4751/3986

36. Madera-Anaya M, Tirado-Amador L, GonzálezMartínez F. Factores relacionados con la empatía en estudiantes de enfermería de la Universidad de Cartagena. Enferm Clin 2016; 26:282-9. Disponible en: https://doi.org/10.1016/j.enfcli.2016.06.004

37. Harris AD, McGregor, JC, Perencevich, EN, Furuno, JP, Zhu J, Peterson DE, \& Finkelstein J. The use and interpretation of quasiexperimental studies in medical informatics. Journal of the American Medical Informatics Association, 2006; 13 (1): 16-23. Doi: https://doi.org/10.1197/jamia.M1749

38. McLean, S. F. Case-Based Learning and its Application in Medical and Health-Care Fields: A Review of Worldwide Literature. Journal of Medical Education and Curricular Development. 2016; (3): 39-49. Doi: https://doi.org/10.4137/JMECD.S20377

39. Ministerio de Salud [Internet]. Plan Decenal de Salud Pública 2012-2021 [Actualizado 12 Dic 2018; Citado 10 Ene 2017]. Disponible en: https://www.minsalud.gov.co/plandecenal/Pa ginas/home2013.aspx

40. Center for Research in Medical Education \& Health Care [internet]. Jefferson Scale of Empathy. Philadelphia University and Tomas Jefferson University [Actualizado 12 Dic 2016; Citado 14 Nov 2017]. Disponible en: http://www.jefferson.edu/university/skmc/res earch/research-medical-education/jeffersonscale-of-empathy.html

41. Quince T, Thiemann P, Benson J, Hyde S. Undergraduate medical students' empathy: current perspectives. Advances in Medical Education and Practice. 2016; 7:443-455. Doi: https://doi.org/10.2147/AMEP.S76800.

42. Leombruni P, Di Lillo M, Miniotti M, Picardi A, Alessandri G, Sica C, et al. Measurement properties and confirmatory factor analysis of the Jefferson Scale of Empathy in Italian medical students. Perspect Med Educ. 2014;
3:419-430. Doi: https://doi: 10.1007/s40037014-0137-9

43. Parra-Ramírez G, Cámara-Vallejos R. Nivel de empatía y factores asociados en estudiantes de medicina. Inv Ed Med. 2016; 24 (6): 221227.

Doi: https://doi.org/10.1016/j.riem.2016.11.001

44. Ozcakir A, Ediz B, Bilgel N. The relationship between subjective well-being and empathy among Turkish medical students. MedEdPublish. 2016; 5(2): 31 Doi: https://doi.org/10.15694/mep.2016.000059

45. Etikan I, Abubakar S, Rukayya -Sunusi R. Comparison of Convenience Sampling and Purposive Sampling. American Journal of Theoretical and Applied Statistics. 2016, 5;(1): 1-4.

Doi:

http://doi.org/10.11648/j.ajtas.20160501.11.

46. DeBono R, Vincenti K, Calleja N. Risk communication: climate change as a humanhealth threat, a survey of public perceptions in Malta, European Journal of Public Health. 2012; 22(1): 144-149. Doi: https://doi.org/10.1093/eurpub/ckq181

47. Dean S, Foureur M, Zaslawski C, Newton-John $T, Y u$ N, Pappas E. The effects of a structured mindfulness program on the development of empathy in healthcare students. NursingPlus Open. 2017;3: 1-5. Doi: http://dx.doi.org/10.1016/j.npls.2017.02.001

48. Hojat, M. Mangione S, Nasca TJ, Rattner S, Erdmann JB, Gonnella JS, Magee M. An empirical study of decline in empathy in medical school. Medical Education.2004; 38(9):934-41.

Doi: https://doi.org/10.1111/j.1365-

2929.2004.01911.x

49. Sinclair S, Norris JM, McConnell SJ, Chochinov HM, Hack TF, Hagen NA, et al. Compassion: a scoping review of the healthcare literature. BMC Palliative Care. 2016; 15(6). Doi: https://doi.org/10.1186/s12904-016-0080-0

50. Tierney S, Seers K, Tutton E, Reeve J. Enabling the flow of compassionate care: a grounded theory study. BMC Health Services Research. 2017; $\quad$ 17:174.

Doi: 
https://doi.org/10.1186/s12913-017-2120-8.

51. Kreslake JM, Sarfaty M, Roser-Renouf C, Leiserowitz AA, Maibach EW. The Critical Roles of Health Professionals in Climate Change Prevention and Preparedness. American Journal of Public Health. 2018;108(Suppl 2): S68-S69. Doi: https://doi.org/10.2105/AJPH.2017.304044.

52. Milfont TL, Wilson MS, Sibley CG. The public's belief in climate change and its human cause are increasing over time. Lötters S, ed. PLoS ONE. 2017;12(3):e0174246. Doi: https://doi.org/10.1371/journal.pone.017424

6.

53. Basu R, Rau R, Pearson D, Malig B. Temperature and Term Low Birth Weight in California. Am J Epidemiol. 2018; 187(11): 2306-2314. Doi: http://doi.org/10.1093/aje/kwy116

54. Sherman GD, Lerner JS, Renshon J, Ma-Kellams C, Joel S. Perceiving others feelings: the importance of personality and social structure. Social Psychological and Personality Science. 2015; 6(5): 559-569. Doi: http//doi.org/10.1177/1948550614567358 